African Crop Science Journal by African Crop Science Society is licensed under a Creative Commons Attribution 3.0 Uganda License. Based on a work at www.ajol.info/ and www.bioline.org.br/cs DOI: https://dx.doi.org/10.4314/acsj.v28i1.20S

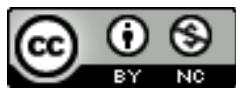

\title{
FAMILIARISME ET PERFORMANCE EN CONTEXTE DE PETITES ET MOYENNES ENTREPRISES FAMILIALES : UNE ÉTUDE EXPLORATOIRE
}

\author{
B. BINWA ${ }^{1,2}$, A. MMENGE ${ }^{1,2}$ et D. GAHUNGU ${ }^{1,2}$ \\ ${ }^{1}$ Faculté des Sciences Économiques et de Gestion, Université du Burundi \\ ${ }^{2}$ Centre Universitaire de Recherche pour le Développement Economique et Social (CURDES)
}

Auteur correspondant: benoitbinwa@gmail.com

\section{RÉSUMÉ}

Cette étude identifie les attributs du familiarisme en contexte de petites et moyennes entreprises familiales (PMEF) et definit la performance telle que perçue par les entrepreneurs familiaux dans un contexte sans marché financier. Appliquant la méthode d'étude de cas par comparaison inter-sites sur la base d'entretiens semi-directifs organisés avec 32 entrepreneurs familiaux de deux villes et de deux cités de province du Nord et du Sud-Kivu, en République Démocratique du Congo, les résultats indiquent que, comparativement aux indicateurs de l'effet famille assortis de travaux antérieurs, l'opérationnalisation de ce construit s'apprête légèrement moins dans le contexte congolais. Pendant ce temps, la performance perçue par les opérateurs économiques s'éloigne considérablement des considérations théoriques.

Mots Clés: Etude de cas, familiarisme, famille, harmonie, performance

\begin{abstract}
Faced with the current economies, characterised by a combination of complex factors, competitiveness of small and medium enterprises (SME) in countries like the Democratic Republic of Congo has become a necessity for the the sustainability of the private sector. In spite of the prevalence of family businesses in several countries in the sub-Saharan Africa and their proven role in developing economies, operational issues of familiarism and performance are not still clearly elucidated in the context of SME family businesses, nor submitted to the stock market. The objective of this study was to elucidate and operationalise the "family effect "and" performance "in the context of SME which are not subjected to the stock market as perceived by family entrepreneurs. Applying the inter-site comparison case study method on the basis of semi-structured interviews organised with 32 family entrepreneurs from two towns and two cities in the provinces of North and South Kivu in the Democratic Republic of Congo, the results indicate that, apart from the deeply modified family effect indicators, the dimensions of the construct are identical to previous works. Meanwhile, the performance perceived by economic operators greatly diverges from theoretical considerations.
\end{abstract}

Key Words: Case study, familiarism, family, harmony, performance 


\section{INTRODUCTION}

Face au paysage des économies actuelles, caractérisé par une combinaison des facteurs complexes (Galibaka et Kangoye, 2014), la compétitivité est devenue une nécessité (Ndjambou et Sassine, 2014; Cao et al., 2017) absolue pour la performance (Diéguez-Soto et al., 2015) et la pérennité du secteur privé (Renaud, 2009). Nonobstant la prévalence des entreprises familiales dans plusieurs pays (Germain, 2009) et leur rôle avéré dans les économies en développement (Pindado et al., 2008), les questions opérationnelles du familiarisme et de la performance ne sont pas encore clairement élucidées en contexte des petites et moyennes entreprises familiales non soumis au marché boursier. Ces difficultés sont consécutives au manque de consensus dans la définition de l'entreprise familiale (Vincencová et al., 2015) et dans la mesure de la performance (Lönnqvist, 2002). Pourtant, l'opérationnalisation d'un construit est postérieure à son cadre conceptuel précis. Tenant compte de l'unicité de l'entreprise familiale (Agbim, 2019), l'effet famille ou familiarisme, désigne les attributs qu'apporte la famille à l'entreprise et qui affecte sa performance (Vallejo-Martos et al., 2016).

Etant donné que la performance est souvent influencée par des questions relatives aux relations familiales (Anantadjaya et al., 2010; Joshi et al., 2019), à l'harmonie dans la famille (Venter et al., 2012) ainsi qu'à la gouvernance familiale (Basco, 2018), cette étude postule que la mise à profit par les familles de leurs ressources idiosyncratiques au sein de l'entreprise familiale, peut constituer une source d'avantages compétitifs (Habbershon et Williams, 1999; Bauweraerts et Colot, 2013) et conduire à la performance de celles-ci.

Cette étude exploratoirepoursuivait deux objectifs complémentaires : (i) identifier les attributs du familiarisme en contexte de petites et moyennes entreprises familiales (PMEF) non soumises au marché financier, et (ii) définir la performance telle que perçue par les entrepreneurs familiaux. Contrairement aux études précédentes qui se soient inscrites dans une logique positivisteet dans un environnement où les marchés finnciers sont développés (Pindado et al., 2008; Suadiye, 2017; Jaziri, 2018), cette étude a adopté une démarche constructivite. A travers les interactions sociales avec les entrepreneurs familiaux de petite et moyenne entreprises répartis dans deux villes et deux cités de la République Démocratique du Congo, l'étude a mis en lumière les contributions de membres familiaux dans la gouvernance de l'entreprise familiale ainsi que les indicateurs fréquemment utilisés pour apprécier la performance réalisée.

Revue de littérature. Les concepts de familiarisme et de performance dans les entreprises familiales ont déjà fait objet d'études antérieures. L'influence de la famille dans le fonctionnement des entreprises familiales est connue sous le vocable« familiarisme ou effet famille». Ces concepts renvoient aux attributs qu'apporte la famille à l'entreprise et qui affectent la performance de celle-ci (Gibb, 2006 ; Kontinen et al., 2012). Autrement dit, il s'agit de la contribution des membres de famille-souvent les plus proches, à travers leurs ressources rares et uniques, au bon fonctionnement de l'entreprise familiale. Par entreprise familiale, l'étude sous-entend, une entreprise tenue, contrôlée et/ou dirigée majoritairement ou totalement par une famille ou un groupe familial, et dont l'objectif en amont reste la satisfaction des besoins familiaux et en aval, la transmission intergénérationnelle. Les membres de famille sont des individus liés par une relation directe (famille nucléaire), indirecte (élargie aux autres membres) ou issus de l'alliance matrimoniale. Toutes les générations de ces différentes catégories peuvent faire leur apparition dans l'entreprise familiale (Arshed et Danson, 2016). L'opérationnalisation la plus célèbre de l'effet famille est empruntée de travaux de Klein et al. (2005). Pour ces auteurs, l'influence de la famille dans 
l'entreprise peut se réaliser à travers trois composantes connues sous le vocable « FPEC (Family-Power, Experience and Culture) ». La dimension « pouvoir » renferme la propriété actionnariale, la participation dans une holding ou dans un cartel, la participation dans la gouvernance ainsi que la participation active à la gestion. La dimension « expérience » renvoie à la succession et renseigne sur la génération qui tient les affaires. La dimension «culture » fait allusion aux valeurs de l'entreprise et de la famille. Cette échelle tient son pesant d'or du fait qu'elle ait défini, de manière holistique, le cadre d'exercice de l'influence des membres familiaux dans l'entreprise familiale. Cependant, l'analyse profonde de ses indicateurs ainsi que certaines sous-dimensions (participation dans le holding) montre que tous les éléments retenus ne sont pas appropriés pour les petites et moyennes entreprises de pays en développement non soumis au marché financier. La plupart d'études postérieures (Sherif et Tahir, 2016 ; Diéguez-Soto et al., 2017), se réfèrent le plus souvent à la dimension pouvoir (Arshed et Danson, 2016) pour étudier les effets de l'implication des membres familiaux. Rares sont les travaux(Chong et al., 2013 ; Gill et Kaur, 2015) qui valorisent l'ensemble des dimensions définies précédemmentpar le FPEC.

S'agissant de la performance, sa mesure n'a jamais fait objet de consensus (Lönnqvist, 2002 ; Gotteland et al., 2007). Les débats sont ouverts entre ceux qui privilégient les mesures subjectives et les partisans des mesures objectives. Cette diversité s'observe davantage lorsque les unités d'enquête concernées par l'étude évoluent dans un marché financier ou de capitaux. A ce titre, Diéguez-Soto et al., (2015) mesurent la performance des entreprises familiales sur base des indicateurs tels que les dettes, l'efficience, la croissance et la profitabilité. Agbim (2019) quant à lui, constate que les mesures les utilisées dans le cadre des entreprises familiales ont trait à la croissance et à la profitabilité. La première dimension réfère au nombre d'employés qualifiés alors que la seconde renvoie à la rentabilité économique et au q de Tobin. Pendant ce temps, Azoury (2010) estime que les indicateurs rationnels de mesure de performance des entreprises familiales renvoient aux critères relatifs à l'augmentation du revenu, à l'augmentation du chiffre d'affaires, à l'augmentation du résultat net, au ratio dette/capitaux, à l'augmentation du taux de rendement des actifs, à l'augmentation du taux de rendement du capital ainsi qu'à l'augmentation de dividendes versés. Faisant suite à cet amalgame, certains chercheurs proposent de tenir compte des spécificités des entreprises familiales ainsi que du contexte de leur environnement (Mahmood et al., 2018). En réponse à cette demande, Williams et al. (2019) ont mis en place une échelle de mesure de la performance basée sur les objectifs idiosyncratiques dévolus aux entreprises familiales. Cette échelle est développée sur base de 21 items répartis en six dimensions représentant également six objectifs : axes famille-entreprise familiale, entreprisecommunauté, stratégique et financier, compensation personnelle, succession, croyances familiales-religion. Malgré sa pertinence du fait qu'elle tienne compte des objectifs poursuivis par les entrepreneurs familiaux, faut-il souligner que certains de ses indicateurs vont la peine d'être clarifiés non seulement du fait que l'échantillon des entreprises sur base duquel était construite l'échelle était constitué des entreprises cotées en bourse, mais égalementdes entreprises familiales et non familiales. Cette échelle renferme surtout certains objectifs non poursuivis par les petites et moyennes entreprises familiales de pays en développement non soumis au marché financier. C'est le cas notamment de la protection de l'environnement dans l'axe dédié à la relation entre l'entreprise et la communauté. Ces arguments justifient la nécessité de définir ces concepts tenant compte des spécificités locales. 


\section{MÉTHODOLOGIE}

L'analyse empirique de cette recherche se basait sur la méthode d'étude de cas (Collerette, 1997) par comparaison inter-sites (Audet et Couteret, 2005) qui était appropriée pour une démarche interprétative (AubinAuger et al., 2008) et compréhensive (Dumez, 2016). Elle a permis de préciser et d'approfondir les connaissances existantes (Trudel et al., 2006), en plus d'une réponse parfaite à la complexité du phénomène étudié (Benchrifa et al., 2017). Cette méthode avait été précédée par une revue théorique fouillée, ayant permis d'aborder le terrain. En même temps, elle laissait une marge de manœuvre aux répondants afin que ce dernier ait la possibilité de faire émerger des situations nouvelles. Cette équivocité lui a conféré à la fois, les caractéristiques des démarches déductives et inductives(Audet et Couteret, 2005). L'enquête a été réalisée du $1^{\mathrm{er}}$ au 21 Octobre 2019 dans les provinces du Nord et Sud-Kivu, en République Démocratique du Congo. Elle s'est déroulée, pour les uns, dans les entreprises ou bureaux de services durant les heures de travail. Pour d'autres, les entretiens ont été réalisés en leurs domiciles sur base d'un rendez-vous. La durée moyenne était de 90 minutes. Chaque fois, nous nous décidions d'arrêter le dialogue après constat de la saturation sémantique. Les cas, constitués des entreprises de petite et moyenne tailles familiales,ont été sélectionnés suivant la richesse de l'information à recueillir. L'étude a ciblé aléatoirement puis par boule de neige 32 dirigeants d'entreprises répartis dans sept secteurs économiques notamment le transport, le commerce, la santé, l'industrie, les dépôts, le pétrole et l'hôtellerie. Le choix de ces secteurs étaitjustifié par le fait que non seulement ils étaient les plus dominants en affaires familiales mais également les acteurs étaientles mieux informés sur les apports directs ou indirects qu'ils recevaient de leurs membres familiaux dans le cadre de la gouvernance courante de leurs entreprises. En même temps, ils étaientoutillés pour donner leur appréciation sur la performance de leurs affaires, sur base des objectifs initialement assignés. La question principale visait de comprendre la contribution de membres familiaux dans le bon fonctionnement des entreprises familiales, et la manière dont cette contribution s'observait concrètement.

Certaines entreprises faisant recours aux travailleurs non familiaux, nous avons été amenés à savoir le comportement de ces derniers, leur attitude ainsi que leur degré d'engagement dans l'entreprise, en présence des ayant-droit. S' agissant de la performance, il était question de raconter dans quelles circonstances ils estiment que les choses vont mieux, et comment cela s'évalue réellement. Etant donné que les cadres théoriques ne sont pas toujours exclusifs et pouvait offrir des explications en partie complémentaires, nous avons fait également recours à des hypothèses rivales plausibles (Dumez, 2011). En effet, pour mieux comprendre les indicateurs de la performance, les opérateurs économiques ont été demandés à décrire des situations de contre-performance. Par la même occasion, il a été question d'indiquer les attitudes qui témoignaientun relâchement des membres familiaux à soutenir l'entreprise. Ces différentes questions ont été définies en étroite articulation avec la partie théorique (Schneider, 2007). Ces débats, en plus de l'observation directe et de l'analyse documentaire (informations fiscales), ont permis l'enrichissement des connaissances. Dumez (2011) affirme que "l'hétérogénéité des sources empiriques dans la recherche qualitative, en garantit l'objectivité ».

Il ressort de ce Tableau 1 que les entreprises concernées par l'étude opèrent majoritairement (44\%) dans le secteur commercial communément appelé commerce général (Khenge et Kinda). En faitce secteur, le plus répandu en RDC, recouvre les entreprises qui vendent à l'état des « produits divers ». Pour faciliter le regroupement, les activités d'habillement, de matériaux de construction, 
TABLEAU 1. Description de l'échantillon

\begin{tabular}{|c|c|c|c|c|c|c|c|}
\hline Secteur d'activité & $\begin{array}{c}\text { Transport } \\
\text { (7) }\end{array}$ & $\begin{array}{l}\text { Commerce } \\
\text { général } \\
(15)\end{array}$ & $\begin{array}{l}\text { Santé } \\
(2)\end{array}$ & $\begin{array}{l}\text { Industrie } \\
\text { (3) }\end{array}$ & $\begin{array}{l}\text { Comptoirs } \\
\text { et dépôts } \\
\text { (2) }\end{array}$ & $\begin{array}{l}\text { Pétrole } \\
\text { (1) }\end{array}$ & $\begin{array}{c}\text { Bars et hôtels } \\
\text { (2) }\end{array}$ \\
\hline Localisation & $\begin{array}{l}B: 4 \\
G: 3 \\
M: 0 \\
U: 0\end{array}$ & $\begin{array}{l}B: 2 \\
G: 3 \\
M: 4 \\
U: 6\end{array}$ & $\begin{array}{l}B: 1 \\
G: 1 \\
M: 0 \\
U: 0\end{array}$ & $\begin{array}{l}B: 1 \\
G: 1 \\
M: 0 \\
U: 1\end{array}$ & $\begin{array}{l}B: 2 \\
G: 0 \\
M: 0 \\
U: 0\end{array}$ & $\begin{array}{l}B: 0 \\
G: 1 \\
M: 0 \\
U: 0\end{array}$ & $\begin{array}{l}B: 1 \\
G: 1 \\
M: 0 \\
U: 0\end{array}$ \\
\hline Ancienneté & $\begin{array}{l}<5 \text { ans : } 2 \\
\text { 5-10ans : } 3 \\
>10 \text { ans : } 2\end{array}$ & $\begin{array}{l}<\text { 5ans : } 3 \\
\text { 5-10ans : } 4 \\
>10 \text { ans : } 7\end{array}$ & $\begin{array}{l}<5 \text { ans : } 0 \\
\text { 5-10ans : } 0 \\
>10 \text { ans : } 2\end{array}$ & $\begin{array}{l}<\text { 5ans : } 0 \\
\text { 5-10ans :1 } \\
>10 \text { ans : } 3\end{array}$ & $\begin{array}{l}<\text { 5ans : } 1 \\
\text { 5-10ans : } 1 \\
>10 \text { ans : } 0\end{array}$ & $\begin{array}{l}<5 \text { ans : } 0 \\
\text { 5-10ans :0 } \\
>10 \text { ans : } 1\end{array}$ & $\begin{array}{l}<5 \text { ans : } 0 \\
\text { 5-10ans : } 1 \\
>10 \text { ans }: 1\end{array}$ \\
\hline Qualité du répondant & $\begin{array}{l}\text { Propr. : } 2 \\
\text { Autres : } 5\end{array}$ & $\begin{array}{l}\text { Propr. : } 11 \\
\text { Autres : } 4\end{array}$ & $\begin{array}{l}\text { Propr. : } 2 \\
\text { Autres : } 0\end{array}$ & $\begin{array}{l}\text { Propr. : } 1 \\
\text { Autres : } 2\end{array}$ & $\begin{array}{l}\text { Propr. : } 1 \\
\text { Autres : } 1\end{array}$ & $\begin{array}{l}\text { Propr. : } 0 \\
\text { Autres : } 1\end{array}$ & $\begin{array}{l}\text { Propr. : } 1 \\
\text { Autres : } 1\end{array}$ \\
\hline Catégorisation & $\begin{array}{l}\mathrm{PE}: 5 \\
\text { ME : } 2 \\
\text { Micro :0 }\end{array}$ & $\begin{array}{l}\mathrm{PE}: 7 \\
\mathrm{ME}: 3 \\
\text { Micro : } 5\end{array}$ & $\begin{array}{l}\text { PE : } 1 \\
\text { ME : } 1 \\
\text { Micro : } 0\end{array}$ & $\begin{array}{l}\mathrm{PE}: 2 \\
\mathrm{ME}: 1 \\
\text { Micro : } 0\end{array}$ & $\begin{array}{l}\text { PE :0 } \\
\text { ME : } 1 \\
\text { Micro : } 1\end{array}$ & $\begin{array}{l}\text { PE :0 } \\
\text { ME }: 1 \\
\text { Micro : } 0\end{array}$ & $\begin{array}{l}\text { PE }: 1 \\
\text { ME }: 1 \\
\text { Micro }: 0\end{array}$ \\
\hline Forme juridique & $\begin{array}{l}\text { E.ind : } 6 \\
\text { Société : } 1\end{array}$ & $\begin{array}{l}\text { E.ind : } 15 \\
\text { Société : } 0\end{array}$ & $\begin{array}{l}\text { E.ind : } 2 \\
\text { Société : } 0\end{array}$ & $\begin{array}{l}\text { E.ind : } 3 \\
\text { Société : } 0\end{array}$ & $\begin{array}{l}\text { E.ind : } 1 \\
\text { Société : } 1\end{array}$ & $\begin{array}{l}\text { E.ind : } 0 \\
\text { Société : } 1\end{array}$ & $\begin{array}{l}\text { E.ind : } 2 \\
\text { Société : } 0\end{array}$ \\
\hline Personnel & $\begin{array}{l}d^{\wedge} 2: 2 \\
3-5: 2 \\
>5: 3\end{array}$ & $\begin{array}{l}d^{\wedge} 2: 9 \\
3-5: 5 \\
>5: 1\end{array}$ & $\begin{array}{l}\mathrm{d}^{\wedge} 2: 1 \\
3-5: 0 \\
>5: 1\end{array}$ & $\begin{array}{l}d^{\wedge} 2: 1 \\
3-5: 0 \\
>5: 2\end{array}$ & $\begin{array}{l}d^{\wedge} 2: 1 \\
3-5: 0 \\
>5: 1\end{array}$ & $\begin{array}{l}d^{\wedge} 2: 0 \\
3-5: 0 \\
>5: 1\end{array}$ & $\begin{array}{l}d^{\wedge} 2: 0 \\
3-5: 2 \\
>5: 0\end{array}$ \\
\hline Sexe & $\begin{array}{l}\text { Homme } 4 \\
\text { Femme : } 3\end{array}$ & $\begin{array}{l}\text { Homme : } 10 \\
\text { Femme : } 6\end{array}$ & $\begin{array}{l}\text { Homme : } 2 \\
\text { Femme :0 }\end{array}$ & $\begin{array}{l}\text { Homme : } 2 \\
\text { Femme : } 1\end{array}$ & $\begin{array}{l}\text { Homme : } 1 \\
\text { Femme : } 0\end{array}$ & $\begin{array}{l}\text { Homme : } 1 \\
\text { Femme : } 0\end{array}$ & $\begin{array}{l}\text { Homme : } 2 \\
\text { Femme : } 0\end{array}$ \\
\hline
\end{tabular}

Notes. Propr : Propriétaire. PE : Petite Entreprise, ME : Moyenne entreprise. E .ind : Entreprise individuelle 
d'alimentation y ont été associées. Entretemps, les deux grandes villes (Bukavu et Goma) occupent seules $66 \%$ de l'échantillon alors que l'enquête a ciblé $50 \%$ des entreprises ayant plus de 10 ans d'existence contre $19 \%$ qui avaient moins de 5 ans. Elle a également interrogé $56 \%$ des propriétaires contre $44 \%$ d'autres acteurs (gérants, comptable, directeur, administrateur), près de $69 \%$ de petites entreprises (et micros d'apparence), et la quasi-totalité (91\%) des entreprises étaient individuelles, la majorité (72\%) employaientau plus cinq agents (mari et femme comptant pour un individu), et constituées de $69 \%$ d'hommes (Tableau 1). Le recueil de données s'était effectué à l'aide d'une grille d'entretien. Les entretiens semi-structurés ont été privilégiés. Cet outil, malgré son caractère chronophage, avait permis d'aborder des sujets plus délicats (Aubin-Auger et al., 2008).

Les interviews ont été faites en français et en swahili selon les circonstances. Celles faites en Swahili ont été soigneusement traduites et transcrites en français de telle sorte que le point de vue réel exprimé par l'interviewé ne soit pas dénaturé. Les opinions des acteurs ont été notées en même temps que les échanges se déroulaient sur le terrain.

De commun accord avec les interviewés, nous avons codé les entreprises suivant les milieux pour garantir l'anonymat. En effet, étant donné que l'enquête ait commencé par la ville de Bukavu (B) passant par Goma (G) et Minova $(\mathrm{M})$ pour se terminer à Uvira (U), les entreprises contactées ont été collées des étiquettes renvoyant à leur milieu respectif, et selon l'ordre de contact. A ce titre, B1 a référé à la première entreprise contactée et située à Bukavu, G14 a référé à la quatorzième entreprise contactée et située à Goma, M23 a été la vingt-troisième entité visitée située à Minova, alors qu'U32 a référé à la dernière entreprise contactée et localisée à Uvira.

L'étude a recouru à l'analyse de contenu thématique des mots à l'aide du logiciel TROPES (VF8.5). Sur base de cette analyse, nous avons procédé au codage manuel (Komis et al., 2013) et à un réarrangement faisant émerger les thèmes principaux (Aubin-Auger et al., 2008). A l'intérieur de chaque concept développé, les varbatims ont été associés pour fin d'illustration. Nous avons été sensibles à des situations qui revêtaient un caractère novateur dans les discours des gens. Par la suite, nous avons procédé par une analyse intra-site et inter-sites. En fait, il s'agissait d'analyser en profondeur et de comprendre la nature d'implication dont il était question ainsi que l'indicateur qui était choisi pour évaluer la performance, et ce, pour chaque entreprise d'un secteur d'activité, puis procéder à une comparaison systématique aux indicateurs d'autres entreprises du même environnement. A l'issu de cet exercice, les indicateurs du familiarisme ainsi que de mesure de performance ont été regroupés. En regroupant ces différents indicateurs par catégories de cas, un ensemble d'attributs uniques à un groupe de cas se dégageait. Nous sommes parvenus à une hétérogénéité d'informations issues des différents groupes homogènes, et avons pu faire émerger des catégories plus fines et plus représentatives des idées énoncées dans les discours des acteurs concernés (Trudel et al., 2006).

\section{RÉSULTATS}

Loubet del Bayle (2000) déclarait qu' : «audelà de la formulation de lois explicatives $d u$ réel, la démarche scientifique comporte l'élaboration de constructions plus ambitieuses que l'on appelle des théories et qui constituent le niveau le plus élevé de l'explication ». $\mathrm{Ce}$ faisant, nous présentons une théorie améliorée de l'effet famille et de mesure de la performance, spécifique aux PMEF (Tableau 2). Celle-ci émane du processus d'abstraction des résultats de terrain (Thiétart, 2014). L'analyse du contenu issue des entretiens révèle que l'effet famille reste un construit tridimensionnel constitué du pouvoir, de l'expérience et de la culture. De même, la performance des PMEF peut s'apprécier à 
TABLEAU 2. Théorisation

\begin{tabular}{|c|c|c|}
\hline Concept & Dimensions & Indicateurs \\
\hline \multirow[t]{3}{*}{ Effet famille } & Pouvoir & $\begin{array}{l}\text { Propriété et contrôle familiaux, structure de gestion duale ou } \\
\text { monale, réunions informelles et formelles }\end{array}$ \\
\hline & Expérience & $\begin{array}{l}\text { Forte implication des enfants et leur insertion dès le bas âge } \\
\text { dans les activités courantes, prestations quasi gratuites, } \\
\text { présence des travailleurs externes, membres familiaux non } \\
\text { présents dans l'entreprise mais actifs }\end{array}$ \\
\hline & Culture & $\begin{array}{l}\text { Soutiens financiers, achats répétitifs, alignement des membres } \\
\text { non familiaux à la vision de l'entreprise }\end{array}$ \\
\hline \multirow[t]{3}{*}{ Performance } & Financière & Ventes, profit, parts de marché, liquidité \\
\hline & Personnelle & $\begin{array}{l}\text { Satisfaction des besoins personnels, résolution facile des petits } \\
\text { problèmes, réalisation des ambitions personnelles, réalisation } \\
\text { de projets individuels jadis inexistants, jouissance d'une estime } \\
\text { communautaire, emplois à la société }\end{array}$ \\
\hline & Familiale & $\begin{array}{l}\text { Satisfaction des besoins familiaux, travail aux membres } \\
\text { familiaux, continuité de l'activité en faveur des générations } \\
\text { futures }\end{array}$ \\
\hline
\end{tabular}

travers les dimensions financière, personnelle et familiale. Le Tableau ci-dessous en fournit des informations utiles.

Familiarisme. Au regard des résultats présentés dans le tableau ci-dessus, l'effet famille dans les PMEF se matérialise à travers l'exercice du pouvoir, l'expérience de parties prenantes et la culture en œuvre au sein de l'entreprise (Tableau 2).

S'agissant de l'exercice du pouvoir, il se manifeste dans la propriété, dans le contrôle et dans la direction. Les statistiques précédentes soutiennent ces résultats du fait que $91 \%$ des entreprises sous étude adoptent une forme individuelle alors que $56 \%$ d'elles sont sous commande de propriétaires à charge $\mathrm{du}$ fonctionnement régulier de leurs entreprises. Ces résultats signifient que la concentration de la propriété est entre les mains des individus et que parmi l'équipe dirigeante ou de gestion, d'autres acteurs (internes et/ou externes) s'y retrouvent : « je suis directeur et membre de famille (B2), je suis comptable et non membre de famille (B5 ». Pour la plupart de situations, le propriétaire dirige et contrôle le bon fonctionnement de l'entreprise. Ceci se constate à travers ces extraits : "...si je ne suis pas ici, c'est ma femme (U31),à part mon épouse, je suis seul (M22)». En plus de la dimension entreprise, les familles se mobilisent de leur manière en multipliant les dialogues et réunions informelles au sujet de l'entreprise, et adoptant des stratégies dans le cercle familial en toute régularité : "dans la famille, on parle seulement entreprise (B4), ils travaillent avec dévouement car il y a un dialogue permanent $(G 13) »$. Ces résultats ont déjà été confirmés par le passédans des recherches de nature causale, aussi bien dans les économies développées, qu'en développement. En effet, sur base d'une étude sur 4958 firmes espagnoles, Diéguez-Soto et al. (2015) montrent que l'implication de la famille dans 
la propriété, dans la gestion et dans la gouvernance a un impact sur la performance de la firme familiale. Ces résultats ont été confirmés en Inde. Ainsi, sur base d'une étude sur les firmes familiales cotées indiennes, Arregle et al. (2019) ont récemment trouvé que l'implication de la famille ainsi que sa vision augmentent la performance financière de firmes. Ces différents résultats s'alignent avec ceux trouvés par Maury (2006) sur un échantillon de 1672 firmes ouest-européennes selon lesquels, les firmes activement contrôlées par la famille affichent une grande profitabilité. En même temps, en Europe, la concentration de la propriété familiale exerce un effet positif sur la valeur de la firme (Pindado et al., 2008) du fait de la présence des membres familiaux dans le directoire ainsi que l'influence du fondateur dans l'entreprise. Cette forte concentration facilite le mécanisme de contrôle interne et profite aux actionnaires minoritaires. Ces résultats ont été confirmés en Egypte (Desoky et Mousa, 2012) et sur 712 firmes malaisiennes cotées (Subramaniam, 2018). Ces différents résultats sont soutenus par Anderson et Reeb (2003) qui affirment que lorsque le CEO est tenu par des membres de familles, la performance est mieux que l'inverse.

Ensuite, la dimension expérience est fortement présente dans les entreprises familiales locales. Ceci se matérialise à travers une forte implication directe et indirecte des membres familiaux présents dans l'entreprise et ceux qui se trouvent en dehors de l'entreprise. Surtout, l'insertion des enfants, successeurs éventuels dans la conduite courante de l'entreprise familiale. Quelques extraits tirés des entretiens sont illustratifs : «...prise de conscience que sans cette activité, il n'y a pas de survie (B3), ces agents dont la majorité est externe, travaillent avec courage même si le payement tarde à venir (M23), engagement énorme de mes enfants, ils font de rotation puis ouvrent à partir de $6 h(U 26)$ ». Sur ce chapitre, un opérateur économique nous a surpris par ces déclarations : "nous sommes riches mais laissons des enfants pauvres. Cette situation s'explique par le fait que la plupart d'opérateurs économiques n'impliquent pas leurs enfants dès le bas âge dans les affaires, afin qu'un jour la relève soit assurée. Je vous assure que ceux qui procèdent actuellement de cette façon leurs affaires évoluent mieux (U32) ». Cette allocution montre que l'implication facilite la réussite, épargne contre « les effets Buddenbrooks » (Dans son roman Les Buddenbrook, Thomas Mann (1901) raconte le déclin d 'une entreprise familiale dont les héritiers dilapident le patrimoine durement acquis par leurs prédécesseurs) (Bégin et al., 2014), surtout, la continuité reste le maitre-mot. Faut-il égalementmentionner que l'intention de travailler dans l'entreprise familiale dépend du degré d'implication des successeurs dans les affaires dès l'âge scolaire (Horèièková et Stasiulis, 2019). Cependant, ces actifs ne sont pas à la portée de toutes les entreprises familiales. Certaines familles en étant dépourvues en amont, des petits sursauts d'orgueils et certaines attitudes discourtoises conduisent au dérapage : "les membres familiaux (élargis) travaillent mal(B8), ...par contre ce sont eux qui me mettent en difficultés (M25)».Des études empiriques font également preuve de différence de performance des entreprises familiales consécutives au différentiel des coûts d'agence, d'actifs ou de passifs familiaux (Gibb, 2006).C'est ainsi que l'engagement et les relations familiales fortes favorisent l'adaptation de la firme familiale et conduit vers sa durabilité (Astrachan, 2009). Au Brésil, il a été trouvé que la performance des entreprises familiales est consécutive à l'identité familiale à travers les bonnes relations d'affaires, à l'organisation interne basée sur la loyauté et à la confiance mutuelle sur base des liens familiaux (Dias et al., 2014).

Enfin, la dimension culture se manifeste à travers la mobilisation des membres familiaux au tours de l'entreprise familiale en procédant par des achats répétés et d'envergure, en portant loin l'image de l'entreprise à travers les sensibilisations et les recommandations, en procédant au financement direct de 
l'entreprise, et surtout en alignant les travailleurs externes dans la vision de l'entreprise. Quelques faits probants sont observés : "dès que tu entres dans cette entreprise, tu deviens comme l'un de nous, on t'initie aux valeurs et normes familiales (B2), la famille renforce mes activités avec des prêts octroyés (M23), les membres familiaux viennent effectuer des gros achats chez moi (5000\$ par exemple) (U26) ».Par le passé, il a été montré que les interactions familiales jouent un rôle majeur dans le développement des entreprises familiales. A ce sujet, insiste James Jr (1999), les relations personnelles, informelles ou explicites sont autant importantes que celles contractuelles, impersonnelles ou explicites dans les entreprises familiales. Ceci veut dire que, dans certaines situations, l'identité familiale ainsi que les liens familiaux sont des ressources qui conditionnent le succès des entreprises familiales plus que les relations contractuelles.Il en résulte que, dans ces entreprises, le système-famille influence directement les comportements du système-entreprise à travers les mécanismes formels et informels. Cette influence se manifeste à travers les langages et les valeurs qui se répandent sur l'ensemble d'acteurs de la firme (Gavetti et al., 2012). Et comme corolaire, le dynamisme familial impacte les comportements en œuvre au sein de l'entreprise (Bégin et al., 2014).Aux Etats-Unis, Burch et al. (2015) avaient constaté que la performance des entreprises familiales était consécutive aux traditionset à l'interdépendance en œuvre au sein des entreprises familiales. Les résultats quasi similaires ont été observés dans les entreprises familiales Russes (Murzina et al., 2018).

Les enseignements à tirer dans cette section sont nombreuses. D'abord, les affaires familiales sont intensément discutées en famille et les réunions se tiennent pour la plupart, sous forme de communication orale, à quelques exceptions près. La division du travail familial se répercute même dans les entreprises, le chef sert de modèle qu'il inculque aux travailleurs d'où qu'ils viennent. Ensuite, malgré le fort engagement des membres familiaux, dans presque toutes les entreprises familiales, les petits comportements déviants s'y observent avec les membres familiaux, et rarement avec les travailleurs externes. Enfin, dans les entreprises familiales de petite taille, le propriétaire et ou sa femme assure le contrôle et la direction. Cependant, d'autres membres familiaux voire non familiaux participent à des postes de responsabilité et de prise de décision dans quelques entreprises naturellement de taille moyenne. Surtout, les conflits successoraux existent. Un opérateur économique de la ville de Bukavu, citant son confrère en séparation avec sa femme, et dont les biens sont revendiqués par chaque partie (père, épouse et enfants), annonce la faillite d'une société de grande renommée et dont le contentieux traine en justice. Sur un autre chapitre, l'esprit associatif n'est pas dans la culture des congolais : "lorsqu'on s'associe avec les autres tout le monde veut s'enrichir sur le dos des autres, les biens deviennent sans maîtres (G13) ; l'autre qui voulait faire plaisir en s'associant avec sa femme est mis en difficulté actuellement (B7). Cette situation s'observe également dans le cadre de cette étude, où les sociétés ne représentent que $9 \%$. C'est ainsi que pour certains opérateurs économiques, en vue d'anticiper tout futur malentendu, et dans le but de maintenir l'harmonie et l'unité familiale, certains choix sont opérés allant notamment au démembrement de la société en différentes unités individuelles familiales : "la société composée jadis de trois membres familiaux depuis 1999 a été dissoute, chacun a pris sa part, celle-ci est une entreprise individuelle depuis 2013 (B2)», voire à l'ouverture des plusieurs segments de vente pour responsabiliser chaque enfant. La Figure 1 cidessous visualise les mots les plus répétés.

Les résultats de cette figure montrent que le familiarisme se perçoit au préalable à travers l'implication des membres de la famille et leurs apports puis sur base de différents services offerts à l'entreprise familiale (Fig. 1). 


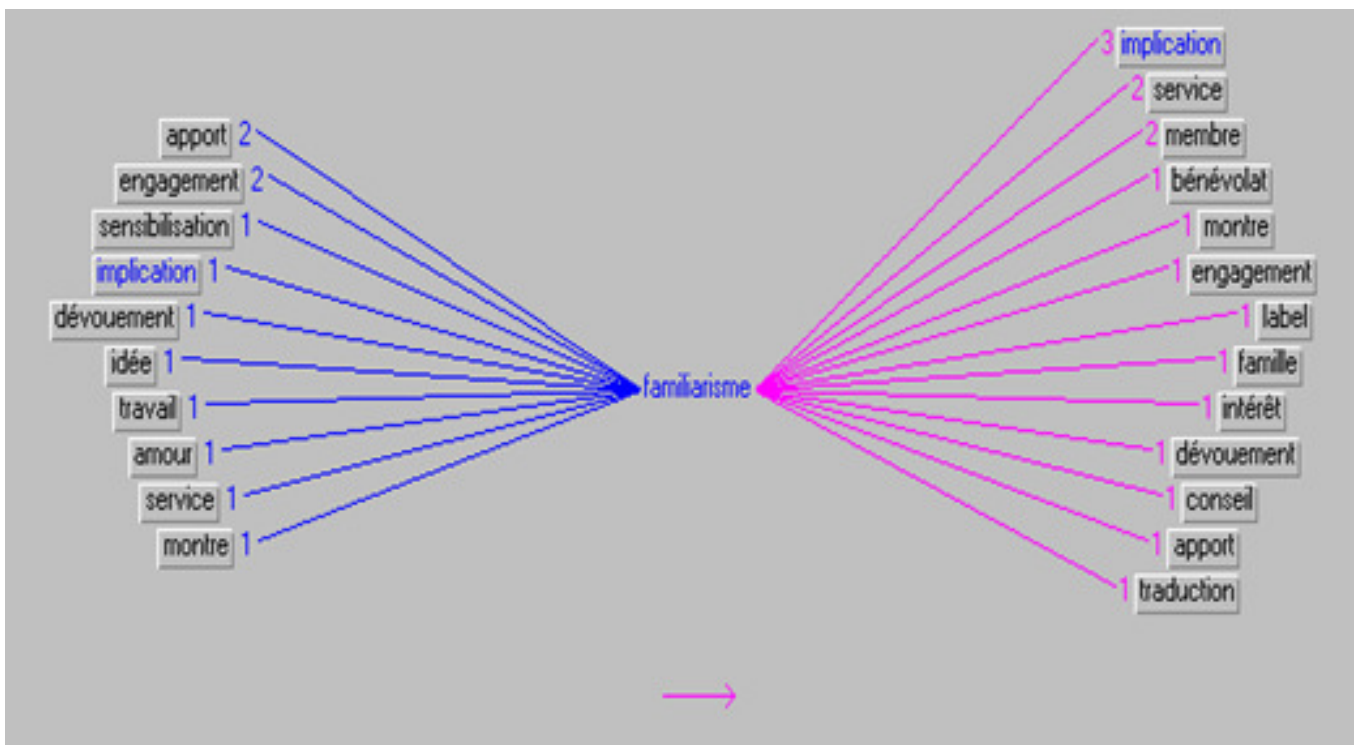

Figure 1. Familiarisme.

Performance perçue. Dans le cadre des entreprises familiales, les indicateurs de performance sont financiers et non financiers (Tableau 2). Les critères financiers se rapportent surtout aux ventes, au profit et à la disponibilité régulière de liquidité. Les critères non financiers ont trait à la satisfaction des besoins de la famille et à celle du propriétaire. Bien plus, lorsque la gestion (administrateur, comptable) est assurée par un expert indépendant, les critères d'évaluation de la performance sont basés sur les indicateurs de bilan et de gestion. Cette attitude se constate également lorsque le propriétaire-dirigeant est instruit dans le domaine des affaires. Cependant, la majorité d'opérateurs économiques se contentent des faits réels, et se passent des calculs comptables sophistiqués. Ces faits sont illustrés par ces déclarations : " pour moi, ce sont les données comptables, mais pour le boss, c'est l'inverse: réaliser ses projets: acheter un véhicule, construire un chantier, avoir besoin de $80000 \$$ pour le dédouanement des marchandises, et se rendre compte que l'argent est là (B10)».

Concernant les indicateurs financiers, la majorité d'opérateurs économiques apprécient la bonne santé de leurs affaires à travers les ventes. A la question de savoir si uniquement les ventes témoignent la performance d'une entreprise, ces derniers indiquent que les prix de vente sont fixés tenant compte de toutes les charges supportées et à supporter; ce qui laisse entendre la notion de profit. En voici les extraits : "augmentation des recettes sur base d'une comparaison des ventes et stabilité de l'entreprise (B7), ventes et rayons achalandés (G17), disponibilité de bons de commande et bénéfice tiré (G15), ventes (no money no job) à travers l'embouteillage des clients et renouvellement des stocks (U27)».De même, parmi les mesures de performance des entreprises familiales recommandées par Azoury (2010) figurent les critères relatifs à l'augmentation du revenu, à l'augmentation du chiffre d'affaires et à l'augmentation du résultat net. Dans la foulée, Utrilla et Torraleja (2012) proposent une mesure optimale de mesure de la performance des entreprises familiales dont la crooissance des ventes figure sur l'axe entreprise. Ces indicateurs ont été qualifiés « distaux » par Ajzen et al. (2016) dans leurs groupes d'attributs de mesure de la performance des entreprises familiales. 
Quant aux critères non financiers, ils se manifestent soit à travers la satisfaction personnelle du propriétaire, soit à travers le bien-être familial. S'agissant de la satisfaction individuelle, l'entrepreneur voit ses ambitions se réaliser, se voit jouir d'une estime communautaire, voit réaliser des investissements jadis absents au paravent, tout en maintenant l'activité de l'entreprise. Ces quelques faits en font large écho : «...lorsque je ne parviens plus à siroter ma bouteille de bière ou déguster de la viande, je comprends que les choses vont mal (B7), fierté d'avoir encadré la jeunesse à travers les stages professionnels et académiques (B1), ...mes investissements : lorsque je passe d'une poule à une chèvre et la résolution des simples problèmes (U28), cas sociaux résolus (G14)».Dans leur étude susmentionnée, Utrilla et Torraleja (2012) avaient également proposé des indicateurs individuels dans l'axe entreprise pour apprécier la performance des entreprises familiales. Ces mêmes idées sont soutenues par Janssen et Taskin (2012).

Enfin, la famille reste le terminal des activités entrepreneuriales familiales. Les entrepreneurs sont tous unanimes que la satisfaction des besoins familiaux est un motif de fierté que l'activité entreprise réalise des bons résultats : «ma famille vit: étudie, mange, soignée; et l'activité continue (B8), satisfaction des besoins familiaux et des travailleurs, notamment l'absence des plaintes (G13), la pérennité de l'activité pour ses futures générations le préoccupe plus (B10) ».L'axe famille de l'échelle de mesure proposée par Utrilla et Torraleja (2012) renferme également des indicateurs se rapportant au bien-être familial et à la succession. De même, sur base d'un échantillon de 12043 entreprises familiales françaises non cotées, Hirigoyen et Ousseini (2017) ont trouvé que les objectifs majeurs des entrepreneurs familiaux sont non économiques et qu'il faille tenir compte de leurs objectifs de pérennité pour mieux comprendre les mécanismes en œuvre dans ces entreprises. Cette position est corroborée par Janssen et Taskin (2012), affirmant que pour l'entrepreneur ou ses membres de famille, la performance correspond plus à un développement familial qu'à une augmentation de la taille de l'entreprise. Ces résultats sont visualisés à travers la Figure ci - après :

Les résultats révèlent que les entrepreneurs familiaux fondent la perception de la performance de leur projet ou exploitation sur les besoins des membres de leurs familles, les ventes et leur satisfaction personnelle (Fig. 2).

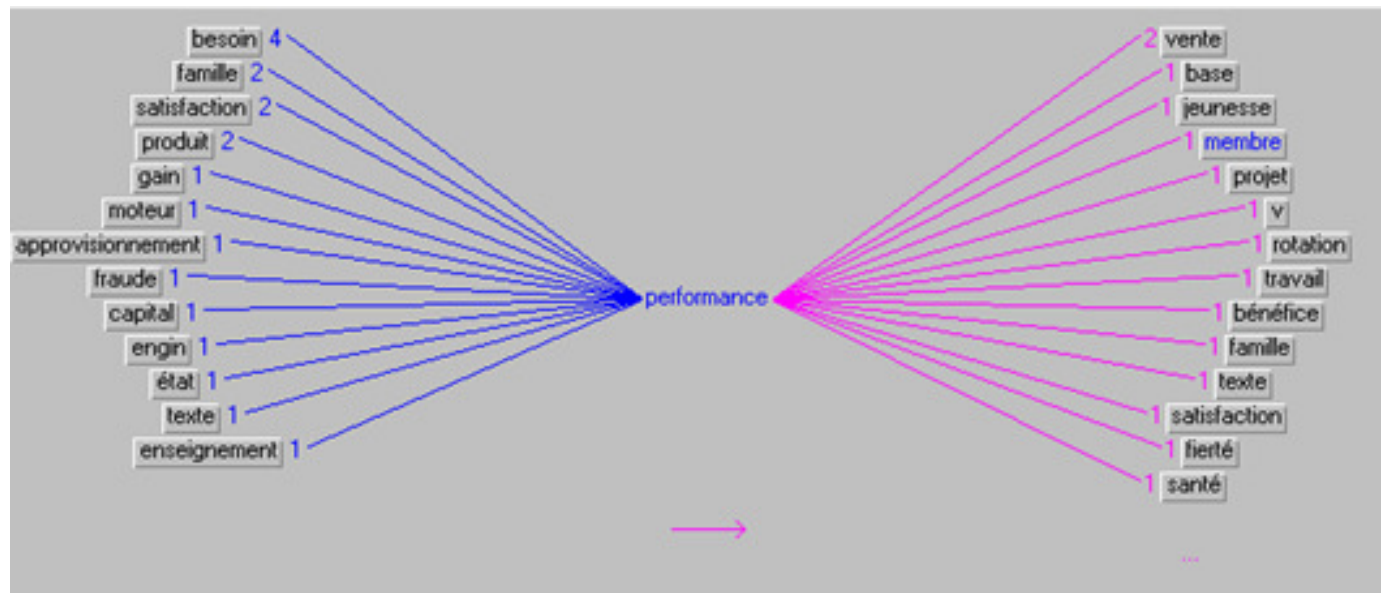

Figure 2. Performance perçue. 


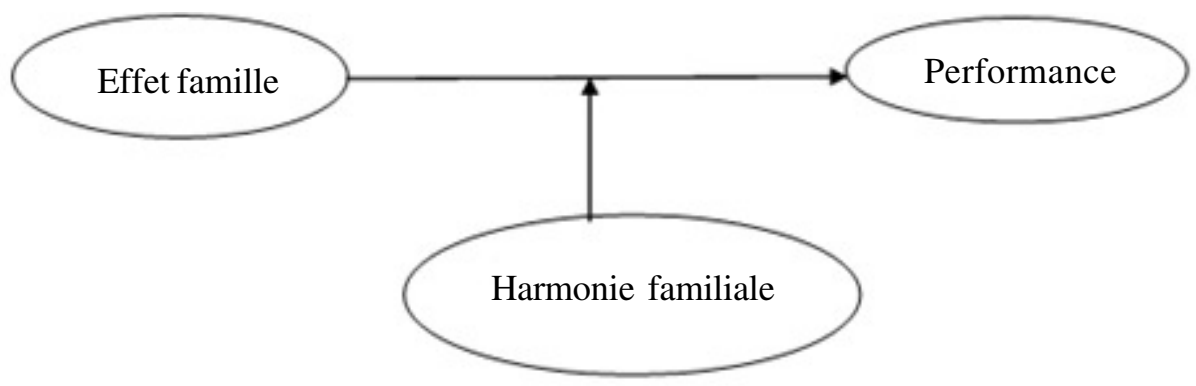

Figure 3. Modèle théorique proposé.

\section{CONCLUSION}

Cette recherche avait pour objectif de définir puis opérationnaliser les concepts «effet famille » et « performance » dans le cadre des entreprises familiales de petite et moyenne taille qui évoluent dans un marché non boursier. La visée étant plus compréhensive qu'explicative, l'approche qualitative a été utilisée pour appréhender le phénomène étudié. Sur base des entretiens avec 32 entrepreneurs familiaux de deux villes et de deux cités de province du nord et du Sud-Kivu, en République Démocratique du Congo, les résultats indiquent que l'opérationnalisation de l'effet famille s'apprête mieux en contexte congolais avec quelques ajustements au niveau des indicateurs. Par contre, la performance perçue par les opérateurs économiques s'éloigne considérablement des considérations théoriques. Etant donné que les familles soient constituées de différents acteurs, à ressources et émotions variées, et que ces dernières se répercutent de manière significative dans l'entreprise familiale, il est impérieux de vérifier empiriquement si les variations observées dans les performances sont consécutives d'une part au degré d'harmonie d'une famille à l'autre, et d'autre part au degré d'implication des différents acteurs dans l'entreprise familiale (Fig. 3).

En effet, l'harmonie de la famille confère à l'entreprise familiale une certaine identité qui facilite l'avantage compétitif (Iaia et al., 2017 ; Murzina et al., 2018). Ceci se justifie par le fait que les familles n'ont pas les mêmes ressources et les mêmes emplois (Gibb, 2006). Entretemps, même les familles qui détiennent suffisamment de ressources sociales (harmonie, engagement) ne peuvent pas le mettre indéfiniment à profit de leur entreprise familiale (Lwango et Cœurderoy, 2011), ce qui laisse présager une forme non linéaire de la relation. Après avoir constaté que l'effet combiné de l'influence familiale et l'innovation conduit à une forte performance des entreprises familiales (Chong et al., 2013), ainsi, postulons-nous que l'interaction entre l'implication de la famille et son harmonie conduisent à une forte performance de cellesci. Ces différentes réflexions feront sans nul doute objet de notre prochain numéro.

\section{RÉFÉRENCES}

Agbim, K.C. 2019. Social networking and the family business performance: A conceptua consideration. Journal of Entrepreneurship, Management and Innovation 15(1):83-122.

Ajzen, M., Rondeaux, G., Pichault, F. et Taskin, L. 2016. Performance et innovation en PME: une relation à questionner. Revue internationale PME 29(2):65-94.

Anantadjaya, S.P., Finardi, A.B. and Nawangwulan, I.M. 2010. Family members et family business activities: A psychological contract? In : 2nd Indonesia International Conference. Innovation, Entrepreneurship and Small Business. School of Business \& Management, 
Institut Teknologi Bandung \& Universitas Multimedia Nusantara. Serpong, Tangerang, Indonesia. pp 1-15

Anderson, R.C. and Reeb, D.M. 2003. Founding family ownership and firm performance: evidence from the S\&P 500. The Journal of Finance 58(3):1301-1328. Arregle, J.-L., Hitt, M.A. and Mari, I. 2019. A missing link in family ûrms' internationalization research: Family structures. Journal of International Business Studies. doi.org/10.1057/s41267019-00213-z.

Arshed, N. and Danson, M. 2016. Enterprise: Concepts and issues. Goodfellow Publishers Ltd. doi: 10.23912/978-1910158-75-3-2873.

Astrachan, J.H. 2009. Using and abusing family business research. DigitalCommons @ Kennesaw StateUniversity.Adviser PersPectives, 20th anniversary.www. familybusinessmagazine.com. Consulté le 10 Novembre 2019.

Aubin-Auger, I., Mercier, A., Imbert, P. et Letrilliart, L. 2008. Introduction à la recherche qualitative. Exercer 84(19): 1-20.

Audet, J. et Couteret, P. 2005. Le coaching entrepreneurial: spécificités et facteurs de succès. Journal of Small Business et Entrepreneurship 18(4): 471-489.

Azoury, N., Salloum, C. et Merheb, M. B. 2010. Les effets non linéaires de la prise de participation sur les performances des entreprises familiales. La Revue des Sciences de Gestion 5:53-61.

Basco, R. 2018. Family Business in Emerging Markets. oup uncorrected proof firstproofs, newgen. Researchgate.

Bauweraerts, J. and Colot, O. 2013. How do family firms deal with the crisis? International Advanced Economics Research 19:313-314.

Bégin, L., Bonnafous-Boucher, M., Chabaud, D. et Fayolle, A. 2014. La longévité des entreprises familiales en question. Revue de l'entrepreneuriat 13(3):11-23.
Benchrifa, H., Asli, A. et Zerrad, J. 2017. Le rôle de la GRH dans la promotion de la dynamique intrapreneuriale. International Journal of Business et Economic Strategy 5(2):91-101.

Burch, G.F., Batchelor, J.H. and Heller, J.J. 2015. Rethinking family business education. Journal of Family Business Management 5(2):277-293.

Cao, S., Salameh, M., Seki, M. and St-Amant, P. 2017. Trends in firm entry and new entrepreneurship in Canada. Canadian Public Policy 43(3):202-220.

Chong, W.Y., Idris, A. and Khin, S. 2013. A research framework for family influence on innovation and business performance.

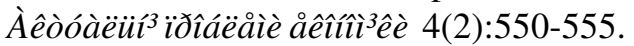

Collerette, P. 1997. L'étude de cas au service de la recherche. Recherche en Soins Infirmiers 50:81-88.

Desoky, A.M. and Mousa, G.A. 2012. Do board ownership and characteristics affect on firm performance? Evidence from Egypt. Global Advanced Research Journal of Economics, Accounting and Finance 1(2):15-32.

Dias, M., Aylmer, R. and Aylmer, M. 2014. Dudalina S/A: Case study on how to overcome succession barriers on a Brazilian family business. Business and Management Review . Available online at http:// www.businessjournalz.org/bmr. special issue - November | 2014

Diéguez-Soto, J., Julio, P. L.-D. and RojoRamírez, A. 2015. Identifying and classifying family businesses. Review of Managerial Science 9(3):603-634.

Dumez, H. 2016. Méthodologie de la recherche qualitative: Les questions clés de la démarche compréhensive. Vuibert. 260pp

Dumez, H. 2011. Qu'est-ce que la recherche qualitative? Le Libellio d'Aegis 7.4-Hiver 47-58.

Galibaka, G. et Kangoye, T. 2014. Améliorer la Compétitivité en Afrique par le Développement des Infrastructures 
Rapport Pays : Sénégal. Série de documents de travail $\mathrm{N}^{\circ}$ 207. Banque africaine de développement, Tunis, Tunisie.

Gavetti, G., Greve, H. R., Levinthal, D. A. and Ocasio, W. 2012. The behavioral theory of the firm: Assessment and prospects. The academy of management annals 6(1):1-40.

Germain, O. 2009. Plaidoyer pour une dynamique entrepreneuriale des entreprises familiales. International Management 2(3): 91-94.

Gibb, W.J. 2006. Examining the "family effect" on firm performance. Family Business Review 19 (4): 253-273.

Gill, S. and Kaur, P. 2015. Family involvement in business and financial performance: A panel data analysis. The Journal for Decision Makers 40(4):395-420.

Gotteland, D., Haon, C. et Gauthier, C. 2007. L'orientation marché: Synthèse et nouvelles directions théoriques. Recherche et Applications en Marketing (French Edition) 22(1):45-59.

Habbershon, T.G. and Williams, M. L. 1999. A resource-based framework for assessing the strategic advantages of family firms. Family Business Review 12(1):1-25.

Hirigoyen, G. and Ousseini, D.M. 2017. Investment Decisions in Unlisted Family Businesses. International Journal of Economics et Management Sciences 6(1):19.

Horèièková, Z. and Stasiulis, N. 2019. Philosophy of economics and management: Youth participation in family business and national economy. Filosofija. Sociologija 30(1).

Iaia, L., Fait, M., Scorrano, P., Cavallo, F. and Maizza, A. 2017. Family vs no family business in wine identity communication. 10th Annual Conference of the EuroMed Academy of the Business. EuroMed Research Business Institute. September 1315 2017. Rome, Italy.

James Jr, H.S. 1999. What can the family contribute to business? Examining contractual relationships. Family Business Review 12(1):61-71.
Janssen, F. et Taskin, L. 2012. Quelles spécificités pour l'étude du changement entrepreneurial ? Revue Interdisciplinaire Management, Homme et Entreprise 3(3): 87-95 .

Jaziri, R. 2018. La pérennité des entreprises familiales après la succession : le cas Tunisien. Revue Africaine de Management 3(1):32-54.

Joshi, M., Sinha, A. K., Dixi, S. and Shukla, B. 2019. Transition Management in a family business: A case of excel transporters. Electronic Journal. doi: 10.2139/ ssrn.2819859.

Khenge, K.M. et Kinda, G.S. 2019. Facteurs organisationnels de la performance sociale des entreprises privées Congolaises: cas des Petites et Moyennes Entreprises de Kinshasa . Researchgate publication.

Klein, S.B., Astrachan, J.H. and Smyrnios, K.X. 2005. The F-PEC scale of family influence: Construction, validation, and further implication for theory. Entrepreneurship Theory and Practice 29(3):321-339.

Komis, V., Depover, C. et Karsenti, T. 2013. L'usage des outils informatiques en analyse des données qualitatives. Researchgate publication.

Kontinen, T., Ojala, A. and Plakoyiannaki, E. 2012. Case studies in family BusineResearch. In: ICSB (Ed.), Proceedings of 57th International Council for Small Business (ICSB) World Conference. Uusi-Seelanti: ICSB.

Lönnqvist, A. 2002. Measurement of intangible assets-an analysis of key concepts. Frontiers of E-business Research 3(27): 275-294.

Loubet del Bayle, J.L. 2000. Initiation aux méthodes des sciences sociales. Paris, L'Harmattan. 350pp

Lwango, A.B. et Cœurderoy, R. 2011. Le capital social de l'entreprise familiale. Une approche empirique de son efficience organisationnelle. Revue Française de Gestion 4(213):109-123. 
Mahmood, N., Barazandeh, F., Eman, N. and Basco, R. 2018. Millennials' family business goals. American University of Sharjah. doi: 10.13140/RG.2.2.35964.85123.

Maury, B. 2006. Family ownership and firm performance: Empirical evidence from Western European corporations. Journal of Corporate Finance 12(2): 321-341.

Murzina, J., Turèeková, N., Mensh, E. and Nakonieczny, J. 2018. Family business and business dynasties: A case study of Russia. Journal of International Studies 11(2): 288-303.

Ndjambou, R. et Sassine, M. 2014. Etude de la relation entre profil du dirigeant, culture et performance des PME gabonaises: clarification des compétences et de la vision. La Revue Gestion et Organisation 6(1):7-19.

Pindado, J., Requejo, I. and Torre, C. D. 2008. Do family firms outperform non-family ones? A panel data analysis of Western European corporations. XVI Foro de Finanzas. Documento de Trabajo 02/08, Spain

Renaud, A. 2009. Les outils d'évaluation de la performance environnementale : audits et indicateurs environnementaux. La place de la dimension européenne dans la comptabilité Contrôle Audit. Strasbourg: CD ROM hlshs-00459153.

Schneider, D.K. 2007. Méthodes qualitatives en sciences sociales. Université de Genève, 54 route des Acacias, $\mathrm{CH}-1227$ Genève.

Sherif, M. and Tahir, M. 2016 . Family Ownership and Firm Performance: the Case of Developed and Emerging Markets. Conference paper. https://www.research gate.net/publication/299445818.
Suadiye, G. 2017. The effects of corporate governance practices on firm performance: empirical evidence from Turkey. CLEAR International Journal of Research in Commerce et Management 8(11):1-8.

Subramaniam, V. 2018. Family ownership and dividend policy: Empirical evidence from Malaysia. International Journal of Business and Management 13(5):1-15.

Thiétart, R.-A. 2014. Méthodes de recherche en management. Paris: 4ème édition. Dunod.

Trudel, L., Simard, C. et Vonarx, N. 2006. $\mathrm{La}$ recherche qualitative est-elle nécessairement exploratoire? Recherches Qualitatives 4(5):38-55.

Utrilla, P. N.-C. and Torraleja, F.A. 2012. Family businesses: How to measure their performance. African Journal of Business Management 6(12):4612-4621.

Vallejo-Martos, M.C., Fuentes-Lombardo, G. and Cano-Rubio, M. 2016. Intangibles in the internationalization of family firms: A source of competitive advantage? ECIC2016-Proceedings of the 8th European Conference on Intellectual Capital .University of Jaen, Jaén, Spain. : https://www.researchgate.net/publication/ 306018355.

Venter, E., Merwe, S.V. and Farrington, S. 2012. The impact of selected stakeholders on family business continuity and family harmony. Southern African Business Review 16(2):69-96.

Williams Jr, I. R., Pieper, T., Kellermanns, F. and Astrachan, J. 2019. Family business goal formation: a literature review and discussion of alternative algorithms. Management Review Quarterly 3(2):1-21. 